\title{
Direct limits of operators in the Hilbert space
}

\author{
Wojciech Mikołajczyk (D)
}

AGH University of Science and Technology, Faculty of Applied Mathematics, Krakow

\begin{abstract}
We present an application of the direct (inductive) limit approach to Toeplitz operators on Segal-Bargmann space. The space corresponds to some analytic functions of infinitely many variables that are square integrable with respect to a Gaussian measure. There are different approaches to such operators that only seem equivalent but lead to different properties of Toeplitz operators. Among the used tools are tensor products, isometric inductive limits and frames.

Keywords: direct limit of operators, Toeplitz operator, Segal-Bargmann space, tensor product of Hilbert spaces, frame

\section{GRANICE INDUKTYWNE OPERATORÓW W PRZESTRZENI HILBERTA}

Streszczenie: Przedstawione zostanie zastosowanie techniki granicy induktywnej do określenia operatorów Toeplitza na przestrzeni Segala-Bargmanna. Przestrzeń ta odpowiada pewnym funkcjom analitycznym nieskończenie wielu zmiennych, które są całkowalne z kwadratem względem pewnej miary Gaussa. Do takich operatorów istnieją różne podejścia, które pozornie są równoważne, jednak prowadzą do różnych własności operatorów. Pośród wykorzystanych metod znajdują się produkty tensorowe, izometryczne granice induktywne oraz kraty (frames). Słowa kluczowe: granica induktywna operatorów, operatory Toeplitza, przestrzeń Segala-Bargmanna, iloczyn tensorowy przestrzeni Hilberta, kraty (frames)
\end{abstract}

https://doi.org/10.7494/978-83-66727-48-9_8 


\section{Isometric inductive limits}

Let $\left\{\mathcal{H}_{n}\right\}$ be a family of non-trivial Hillbert spaces, indexed by natural numbers $n \in \mathbb{H}$. Suppose that for $n \leq m$ we have isometric embeddings $J_{n}^{m}$ of $\mathcal{H}_{n}$ into $\mathcal{H}_{m}$ such that

$$
J_{n}^{n}:=\mathrm{id}_{\mathcal{H}_{n}},
$$$$
J_{n}^{m}=J_{k}^{m} \circ J_{n}^{k} \text { for } n \leq k \leq m .
$$

Then the pair $\left\langle\mathcal{H}_{n}, J_{n}^{m}\right\rangle$ is called an isometric direct system. Denote by $\lim _{\rightarrow} \rightarrow \mathcal{H}_{n}$ the direct limit of this system with $J_{n}$ denoting the isometric embeddings of $\mathcal{H}_{n}$ into $\lim _{\rightarrow} \mathcal{H}_{n}$. These embeddings are related to $J_{n}^{m}$ by the following equalities

$$
J_{n}=J_{m} \circ J_{n}^{m} \text { for all } n \leq m \text {. }
$$

Recall that due to the isometry of the embeddings $J_{n}^{m}$ one can identify $J_{n}^{m}\left(\mathcal{H}_{n}\right)$ with some nested sequence of subspaces $J_{n}\left(\mathcal{H}_{n}\right)$ of an inner product space $\bigcup_{n=1}^{\infty} J_{n}\left(\mathcal{H}_{n}\right)$ and the direct $\operatorname{limit}_{\lim _{\rightarrow}} \mathcal{H}_{n}$ is the completion of this union of subspaces, becoming a Hilbert space, in which this union is dense.

Let us consider a sequence of self-adjoint operators $A_{n}$ defined on dense domains $\mathcal{D}_{n} \subset \mathcal{H}_{n}$. For $n \leq m$ we suppose that by $\left.J_{n}^{m}\left(\mathcal{D}_{n}\right) \subset \mathcal{D}_{m}\right)$. The resulting limit operator will be considered on the set $D_{\infty} \subset \lim _{\rightarrow} \mathcal{H}_{n}$ defined by

$$
\mathcal{D}_{\infty}:=\bigcup_{k \in \mathbb{N}} J_{k}\left(\mathcal{D}_{k}\right)
$$

We say that the sequence $\left\{A_{n}\right\}$ of operators converges strongly and uniformly if for any $\varepsilon, \delta>0$ there is a number $N$ such that for any $N \leq n<m$ and for $J_{n} \varphi \in \mathcal{D}_{\infty}$ the condition $\left\|J_{n} \varphi\right\|<\delta$ implies

$$
\left\|\left(A_{m} J_{n}^{m}-J_{n}^{m} A_{n}\right) \varphi\right\|<\varepsilon .
$$

The following results of (Marčenko 1974, 1975), provide sufficient conditions for the existence and self-adjointness of the limit for this sequence of operators.

Theorem 1. If the sequence of operators $\left\{A_{n}\right\}$ converges strongly and uniformly on the vectors from $\mathcal{D}_{\infty}$, then the operator $A$ given by the equality

$$
A J_{n} \varphi=\lim _{m \rightarrow \infty} J_{m} A_{m} J_{n}^{m} \varphi
$$

for $J_{n} \varphi \in \mathcal{D}_{\infty}$ is well defined and is essentially self-adjoint on $\mathcal{D}_{\infty}$. 
Recall that a densely defined linear operator is essentially self-adjoint, if it is closable (its adjoint has a dense domain) and the closure of this operator is self-adjoint.

For practical use we have more convenient criterion, namely:

Theorem 2. Suppose that for any $\varepsilon>0$ there exists a number $N$ such that for all $N \leq n<m$ and for any $\varphi \in \mathcal{D}_{n}$ the following inequalities hold:

$$
\left\|\left(A_{m} J_{n}^{m}-J_{n}^{m} A_{n}\right) \varphi\right\| \leq \varepsilon\left(\|\varphi\|+\left\|A_{m} J_{n}^{m} \varphi\right\|+\left\|A_{n} \varphi\right\|\right) .
$$

Then the operator $A$ defined by (1) is essentially self-adjoint on $\mathcal{D}_{\infty}$.

These theorems were generalized for sequences of densely defined closable operators (not necessarily self-adjoint) by Janas (1995) in the following way. Let $\mathcal{D}_{n}^{*} \subset \mathcal{H}_{n}$ denote the domain of the adjoint $A_{n}^{*}$ for our operator $A_{n}$. We suppose that the above domains satisfy the following condition for any $n \leq m$ :

$$
J_{n}^{m}\left(\mathcal{D}_{n}\right) \subset \mathcal{D}_{m}, J_{n}^{m}\left(\mathcal{D}_{n}^{*}\right) \subset \mathcal{D}_{m}^{*}
$$

Theorem 3. Let $\left\{A_{n}\right\}$ be a sequence of densely defined closable operators in $\mathcal{H}_{n}$ satisfying (2). Assume that for any $\varepsilon>0$ there exists number $N$ such that for every $N \leq n<m$ and any $\varphi \in \mathcal{D}_{n}, \psi \in \mathcal{D}_{n}^{*}$ we have

$$
\begin{aligned}
& \left\|\left(A_{m} J_{n}^{m}-J_{n}^{m} A_{n}\right) \varphi\right\| \leq \varepsilon\left(\|\varphi\|+\left\|A_{m} J_{n}^{m} \varphi\right\|+\left\|A_{n} \varphi\right\|\right), \\
& \left\|\left(A_{m}^{*} J_{n}^{m}-J_{n}^{m} A_{n}^{*}\right) \psi\right\| \leq \varepsilon\left(\|\psi\|+\left\|A_{m}^{*} J_{n}^{m} \psi\right\|+\left\|A_{n}^{*} \psi\right\|\right) .
\end{aligned}
$$

Then the formula

$$
A J_{n} \varphi=\lim _{m \rightarrow \infty} J_{m} A_{m} J_{n}^{m} \varphi
$$

defines a closable densely defined operator $A$ on $\mathcal{D}_{\infty}$.

Moreover, for any limit $J_{n} \psi \subset \mathcal{D}_{\infty}^{*}$ the limit

$$
B J_{n} \psi=\lim _{m \rightarrow \infty} J_{m} A_{m}^{*} J_{n}^{m} \psi
$$

exists and $B \subset A^{*}$.

\section{Frames in tensor product spaces}

We will be concerned with Hilbert spaces endowed with frames. These objects are in some cases more convenient to use than orthonormal bases. Let us recall that 
a frame in a Hilbert space $\mathcal{H}$ is a sequence of vectors $\mathcal{F}:=\left\{f_{n}\right\}$, where $f_{n} \in \mathcal{H}$, for which there exist constants $0<a \leq b<\infty$ such that

$$
a\|h\|^{2} \leq \sum_{k \in \mathbb{N}}\left|\left\langle h, f_{k}\right\rangle\right|^{2} \leq b\|h\|^{2} \text { for all } h \in \mathcal{H}
$$

The constants $a, b$ are called the upper and lower frame bounds. With the frame $\mathcal{F}$ there is associated an operator $S$ called the frame operator defined by the following equality

$$
S h=\sum_{k \in \mathbb{N}}\left\langle h, f_{k}\right\rangle f_{k} \text { for all } h \in \mathcal{H}
$$

The inner products $\left\langle h, f_{k}\right\rangle$ are called the frame coefficients of the vector $h$. The series defining this operator $S$ converges unconditionally because any frame is a Bessel sequence, which means that the frame coefficients of any $h \in \mathcal{H}$ are square summable and their $l^{2}$-norm is bounded by some multiple of $\|h\|$.

Let $\mathcal{G}=\left\{g_{n}\right\}$ be a frame with bounds $0<c \leq d<\infty$ for the Hilbert space $\mathcal{H}$. If for any $h \in \mathcal{H}$ we have

$$
h=\sum_{k \in \mathbb{N}}\left\langle h, g_{k}\right\rangle f_{k}=\sum_{k \in \mathbb{N}}\left\langle h, f_{k}\right\rangle g_{k}
$$

then we say that the frame $\mathcal{G}$ is a dual frame to $\mathcal{F}$ and (5) is called the reconstruction formula. Dual frames are not unique in general. Since the frame operator $S$ is invertible, the set $\mathcal{F}^{-1}=\left\{S^{-1} f_{n}\right\}$ is also a frame with bounds $0<b^{-1} \leq a^{-1}<\infty$, called the canonical dual frame for $\mathcal{F}$. From this we see that for any frame there exists at least one dual frame.

If the Hilbert space $\mathcal{H}$ is embedded in another Hilbert space $\mathcal{K}$, then the right-hand side of the reconstruction formula (5) for any pair of dual frames in $\mathcal{H}$ defines an orthogonal projection from $\mathcal{K}$ to $\mathcal{H}$. Namely, let $\mathcal{F}, \mathcal{G}$ be a mutually dual pair of frames in $\mathcal{H}$. Then we denote by $P$ an operator defined by the right-hand side of (5). For $x, y \in \mathcal{K}$

$$
\begin{aligned}
\langle P x, y\rangle & =\left\langle\sum_{n \in \mathbb{N}}\left\langle x, g_{n}\right\rangle f_{n}, y\right\rangle=\sum_{n \in \mathbb{N}}\left\langle x, g_{n}\right\rangle\left\langle f_{n}, y\right\rangle= \\
& =\left\langle x, \sum_{n \in \mathbb{N}}\left\langle y, f_{n}\right\rangle g_{n}\right\rangle=\left\langle x, \sum_{n \in \mathbb{N}}\left\langle y, g_{n}\right\rangle f_{n}\right\rangle=\langle x, P y\rangle, \\
P^{2} x= & \sum_{n \in \mathbb{N}}\left\langle P x, g_{n}\right\rangle f_{n}=\sum_{n \in \mathbb{N}}\left\langle\sum_{k \in \mathbb{N}}\left\langle x, g_{k}\right\rangle f_{k}, g_{n}\right\rangle f_{n}= \\
= & \sum_{n \in \mathbb{N}} \sum_{k \in \mathbb{N}}\left\langle x, g_{k}\right\rangle\left\langle f_{k}, g_{n}\right\rangle f_{n}=\sum_{k \in \mathbb{N}}\left\langle x, g_{k}\right\rangle \sum_{n \in \mathbb{N}}\left\langle f_{k}, g_{n}\right\rangle f_{n} \doteq \sum_{k \in \mathbb{N}}\left\langle x, g_{k}\right\rangle f_{k}=P x .
\end{aligned}
$$


The equality $\doteq$ holds because $f_{k} \in \mathcal{H}$ and we use the reconstruction formula (5). Since these equalities hold, we conclude that $P=P^{2}=P^{*}$ so $P$ is an orthogonal projection (from $\mathcal{K}$ to $\mathcal{H}$ ).

For further references on frames one may consult Christensen (2008).

Definition 4. If the sequences of frame bounds $a_{n}, b_{n}$ for a family of frames $\left\{\mathcal{F}_{n}\right\}$ in Hilbert spaces $\mathcal{H}_{n}$ satisfy

$$
0<\lim _{n \rightarrow \infty} a_{n} \leq \lim _{n \rightarrow \infty} b_{n}<\infty,
$$

then we call $\left\{\mathcal{F}_{n}\right\}$ an admissible family of frames.

For every $n \in \mathbb{N}$, let $\mathcal{G}_{n}$ be a dual frame to $\mathcal{F}_{n}$ with bounds $0<c_{n} \leq d_{n}<\infty$. If $\left\{\mathcal{F}_{n}\right\}$ is admissible family of frames then exists at least one family $\left\{G_{n}\right\}$ of dual frames which is also admissible. For example, if $\mathcal{G}_{n}=\mathcal{F}_{n}^{-1}$ is the canonical dual frame to $\mathcal{F}_{n}$ then $\left\{\mathcal{F}_{n}^{-1}\right\}$ is admissible because the following inequalities hold

$$
0<\lim _{n \rightarrow \infty} b_{n}^{-1} \leq \lim _{n \rightarrow \infty} a_{n}^{-1}<\infty .
$$

From now on we will consider the following types of Hilbert space families.

Let $\left\{\mathcal{H}_{n}\right\}$ be a sequence of non-trivial Hilbert spaces. The tensor product of finite amount of $\mathcal{H}_{n}$ spaces (say, of $m$ spaces) will be denoted by $\mathcal{H}_{[m]}$, namely

$$
\mathcal{H}_{[m]}:=\bigotimes_{k=1}^{m} \mathcal{H}_{k}
$$

If we fix an arbitrary sequence of norm one vectors $e_{n} \in \mathcal{H}_{n}$ then we can define isometric embeddings

$$
\begin{aligned}
& J_{n}^{n}:=\operatorname{id}_{\mathcal{H}_{[n]}}, \\
& J_{n}^{m}: \mathscr{H}_{[n]} \ni h \rightarrow h \otimes \bigotimes_{k=n+1}^{m} e_{k} \in \mathcal{H}_{[m]} \text { for all } n<m .
\end{aligned}
$$

In a natural way the pair $\left\langle\mathcal{H}_{[n]}, J_{n}^{m}\right\rangle$ forms a direct system with direct limit denoted by $\lim _{\rightarrow} \mathcal{H}_{[n]}$. The isometric embeddings of $\mathcal{H}_{[n]}$ into $\lim _{\rightarrow} \mathcal{H}_{[n]}$ are given by

$$
J_{n}: \mathcal{H}_{[n]} \ni h \rightarrow h \otimes \bigotimes_{k=n+1}^{\infty} e_{k} \in \lim _{\rightarrow} \mathcal{H}_{[n]} .
$$


It will be convenient to use the notation $\mathcal{H}_{\infty}$ for the obtained direct limit space. Namely, let

$$
\mathcal{H}_{\infty}:=\lim _{\rightarrow} \mathcal{H}_{[n]}
$$

Note that this is not the same as the notion of infinite tensor product $\bigotimes_{k=n+1}^{\infty} \mathcal{H}_{k}$. The latter space can be defined in two inequivalent ways and the differences between von Neumann's definition and the one used by Berezianskii are discussed in Rudol (1992).

Now we use Theorem 2 to construct a frame in $\mathcal{H}_{\infty}$ from an admissible family of frames in $\mathcal{H}_{[n]}$.

The next two lemmas will be useful in construction of frames by tensor products.

Lemma 5. Let $\mathcal{F}_{i}$ be a frame in Hilbert space $\mathcal{H}_{i}$ with bounds $0<a_{i} \leq b_{i}<\infty$ for $i=1,2$ then

$$
\mathcal{F}_{1} \otimes \mathcal{F}_{2}:=\left\{f_{n} \otimes g_{k}: f_{n} \in \mathcal{F}_{1}, g_{k} \in \mathcal{F}_{2}, n, k \in \mathbb{N}\right\}
$$

are the frame in the Hilbert space $\mathcal{H}_{1} \otimes \mathcal{H}_{2}$ with bounds $0<a_{1} a_{2} \leq b_{1} b_{2}<\infty$.

Proof. It is sufficient to show frame inequalities (3) for tensors of the form $h=h_{1} \otimes h_{2} \in \mathcal{H}_{1} \otimes \mathcal{H}_{2}$. For $f_{n} \in \mathcal{F}_{1}, g_{k} \in \mathcal{F}_{2}$ we have

$$
\begin{aligned}
& \sum_{(n, k) \in \mathbb{N}^{2}}\left|\left\langle h, f_{n} \otimes g_{k}\right\rangle\right|^{2}=\sum_{(n, k) \in \mathbb{N}^{2}}\left|\left\langle h_{1} \otimes h_{2}, f_{n} \otimes g_{k}\right\rangle\right|^{2}= \\
& =\sum_{n \in \mathbb{N}} \sum_{k \in \mathbb{N}}\left|\left\langle h_{1}, f_{n}\right\rangle\right|^{2}\left|\left\langle h_{2}, g_{k}\right\rangle\right|^{2}=\sum_{n \in \mathbb{N}}\left|\left\langle h_{1}, f_{n}\right\rangle\right|^{2} \sum_{k \in \mathbb{N}}\left|\left\langle h_{2}, g_{k}\right\rangle\right|^{2} .
\end{aligned}
$$

In the next step we estimate this from above

$$
\begin{aligned}
& \sum_{n \in \mathbb{N}}\left|\left\langle h_{1}, f_{n}\right\rangle\right|^{2} \sum_{k \in \mathbb{N}}\left|\left\langle h_{2}, g_{k}\right\rangle\right|^{2} \leq b_{1}\left\|h_{1}\right\|^{2} \cdot b_{2}\left\|h_{2}\right\|^{2}= \\
& =b_{1} b_{2}\left\|h_{1} \otimes h_{2}\right\|^{2}=b_{1} b_{2}\|h\|^{2} .
\end{aligned}
$$

In a similar way we estimate from below, so the constants $0<a_{1} a_{2} \leq b_{1} b_{2}<\infty$ are the frame bounds for $\mathcal{F}_{1} \otimes \mathcal{F}_{2}$.

Lemma 6. If $S_{i}$ is the frame operator for the frame $\mathcal{F}_{i}, i=1,2$, then the operator $S_{1} \otimes S_{2}$ is the frame operator for $\mathcal{F}_{1} \otimes \mathcal{F}_{2}$. 
Proof. For the proof we show the equality (4) for tensors $h=h_{1} \otimes h_{2} \in \mathcal{H}_{1} \otimes \mathcal{H}_{2}$. Let us denote by $S$ the frame operator for the frame $\mathcal{F}_{1} \otimes \mathcal{F}_{2}$.

$$
\begin{aligned}
S h & =\sum_{(n, k) \in \mathbb{N}^{2}}\left\langle h, f_{n} \otimes g_{k}\right\rangle f_{n} \otimes g_{k}=\sum_{(n, k) \in \mathbb{N}^{2}}\left\langle h_{1} \otimes h_{2}, f_{n} \otimes g_{k}\right\rangle f_{n} \otimes g_{k}= \\
& =\sum_{n \in \mathbb{N}} \sum_{k \in \mathbb{N}}\left\langle h_{1}, f_{n}\right\rangle\left\langle h_{2}, g_{k}\right\rangle f_{n} \otimes g_{k}=\sum_{n \in \mathbb{N}}\left\langle h_{1}, f_{n}\right\rangle f_{n} \otimes \sum_{k \in \mathbb{N}}\left\langle h_{2}, g_{k}\right\rangle g_{k}= \\
& =S_{1} h_{1} \otimes S_{2} h_{2}=S_{1} \otimes S_{2}\left(h_{1} \otimes h_{2}\right)=S_{1} \otimes S_{2} h .
\end{aligned}
$$

So, we have $S=S_{1} \otimes S_{2}$.

Let $\left\{\mathcal{F}_{n}\right\}$ be family of frames, where $\mathcal{F}_{n}$ is a frame for a space $\mathcal{H}_{n}$ with frame bounds $0<a_{n} \leq b_{n}<\infty$. If the frame bounds satisfy the following condition

$$
0<\prod_{n \in \mathbb{N}} a_{n} \leq \prod_{n \in \mathbb{N}} b_{n}<\infty
$$

then we can construct an admissible family of frames by taking tensor products in the following way.

Let us denote by $\mathcal{F}_{[n]}$ the tensor product of the first $n$ frames,

$$
\mathcal{F}_{[n]}:=\bigotimes_{k=1}^{n} \mathcal{F}_{k}=\left\{f_{p}=\bigotimes_{k=1}^{n} f_{p_{k}}: f_{p_{k}} \in \mathcal{F}_{k}, \boldsymbol{p}=\left(p_{1}, \ldots, p_{n}\right) \in \mathbb{N}^{n}\right\}
$$

After some easy calculations (using Lemma 5) we notice that $\mathcal{F}_{[n]}$ form a frame for the space $\mathcal{H}_{[n]}$ with frame bounds $0<\bigsqcup_{n \in \mathbb{N}} a_{n} \leq \coprod_{n \in \mathbb{N}} b_{n}<\infty$. The frame operator for $\mathcal{F}_{[n]}$ is of the form $S_{[n]}:=\bigotimes_{k=1}^{n} S_{k}$.

Theorem 7. If $\left\{\mathcal{F}_{n}\right\}$ is an admissible family of tensor products of frames then for every $\varepsilon>0$ there exists a number $N$ such that for all $N \leq n<m$ and any $h \in \mathcal{H}_{[n]}$ we have

$$
\left\|\left(S_{[m]} J_{n}^{m}-J_{n}^{m} S_{[n]}\right) h\right\| \leq \varepsilon\left(\|h\|+\left\|S_{[m]} J_{n}^{m} h\right\|+\left\|S_{[n]} h\right\|\right)
$$

and the operator $S$ given by

$$
S J_{n} h=\lim _{m \rightarrow \infty} J_{m} S_{[m]} J_{n}^{m} h
$$

for $J_{n} h \in \mathcal{H}_{\infty}$ (in the notation (7)) is well defined and is essentially self-adjoint on $\mathcal{H}_{\infty}$. 
Proof. First, we notice that domain of $S_{[n]}$ is the whole $\mathcal{H}_{[n]}$, so the condition $J_{n}^{m} \mathcal{D}\left(S_{[n]}\right) \subset \mathcal{D}\left(S_{[m]}\right)$ for all $n<m$ is satisfied.

For the convenience of the reader, we will introduce several notations.

$$
e_{\{n, m\}}:=\bigotimes_{k=n+1}^{m} e_{k}, \quad S_{\{n, m\}}:=\bigotimes_{k=n+1}^{m} S_{k},
$$

$\mathbf{k}:=(\mathbf{a}, \mathbf{b}) \in \mathbb{N}^{n} \times \mathbb{N}^{m-n}$.

Let be $h \in \mathcal{H}_{[n]}$. First for $n<m$ we write some useful equality.

$$
\begin{aligned}
S_{[m]} J_{n}^{m} h & =S_{[m]}\left(h \otimes e_{\{n, m\}}\right)=\sum_{\mathbf{k} \in \mathbb{N}^{m}}\left\langle h \otimes e_{\{n, m\}}, f_{\mathbf{k}}\right\rangle_{m} f_{\mathbf{k}}= \\
& =\sum_{\mathbf{a} \in \mathbb{N}^{n}} \sum_{\mathbf{b} \in \mathbb{N}^{m-n}}\left\langle h, f_{\mathbf{a}}\right\rangle_{n}\left\langle e_{\{n, m\}}, f_{\mathbf{b}}\right\rangle_{m-n} f_{\mathbf{a}} \otimes f_{\mathbf{b}}= \\
& =\left(\sum_{\mathbf{a} \in \mathbb{N}^{n}}\left\langle h, f_{\mathbf{a}}\right\rangle_{n} f_{\mathbf{a}}\right) \otimes\left(\sum_{\mathbf{b} \in \mathbb{N}^{m-n}}\left\langle e_{\{n, m\}}, f_{\mathbf{b}}\right\rangle_{m-n} f_{\mathbf{b}}\right)= \\
& =\left(S_{[n]} h\right) \otimes\left(S_{\{n, m\}} e_{\{n, m\}}\right) .
\end{aligned}
$$

Now we turn to the key estimate

$$
\begin{aligned}
& \left\|\left(S_{[m]} J_{n}^{m}-J_{n}^{m} S_{[n]}\right) h\right\|=\left\|\left(S_{[n]} h\right) \otimes\left(S_{\{n, m\}} e_{\{n, m\}}\right)-\left(S_{[n]} h\right) \otimes e_{\{n, m\}}\right\|= \\
& \quad=\left\|\left(S_{[n]} h\right) \otimes\left(S_{\{n, m\}} e_{\{n, m\}}-e_{\{n, m\}}\right)\right\|= \\
& \quad=\left\|S_{[n]} h\right\|\left\|\left(S_{\{n, m\}}-I\right) e_{\{n, m\}}\right\| \dot{\leq}\left\|S_{[n]} h\right\|\left|\prod_{k=n+1}^{m} b_{k}-1\right|\left\|e_{\{n, m\}}\right\| \ddot{\leq}\left\|S_{[n]} h\right\| \varepsilon .
\end{aligned}
$$

The inequality ( $\dot{)})$ is due to the fact that $S_{\{n, m\}}$ is a frame operator for the frame $\bigotimes_{k=n+1}^{m} \mathcal{F}_{k}$ with upper bound equal $\prod_{k=n+1}^{m} b_{k}$. And the Cauchy condition for infinite product gives us inequality $(\ddot{\Xi})$. Hence the proof is finished by invoking Theorem 2 .

Let $\mathcal{G}_{n}$ denote a dual frame to $\mathcal{F}_{n}$ with bounds $0<c_{n} \leq d_{n}<\infty$ such that the family $\left\{\mathcal{G}_{[n]}=\bigotimes_{k=1}^{n} \mathcal{G}_{k}\right\}$ is admissible. For the next theorem we assume that each Hilbert space $\mathcal{H}_{n}$ is embedded in some Hilbert space $\mathcal{K}_{n}$ and we denote by $P_{n}$ the 
orthogonal projection defined by the reconstruction formula (5). We introduce the following additional notations

$$
\begin{aligned}
& \mathcal{G}_{\{n, m\}}:=\bigotimes_{k=n+1}^{m} \mathcal{G}_{k}, \quad \mathcal{K}_{[n]}:=\bigotimes_{k=1}^{n} \mathcal{K}_{k}, \quad \mathcal{K}_{\{n, m\}}:=\bigotimes_{k=n+1}^{m} \mathcal{K}_{k}, \\
& P_{[n]}:=\bigotimes_{k=1}^{n} P_{k}, \quad P_{\{n, m\}}:=\bigotimes_{k=n+1}^{m} P_{k} .
\end{aligned}
$$

Theorem 8. If $\left\{\mathcal{F}_{[n]}\right\},\left\{\mathcal{G}_{[n]}\right\}$ are admissible and dual families of frames, whose bounds satisfy (8) then for every $\varepsilon>0$ there exists a number $N$ such that for every $N \leq n<m$ and $x \in \mathcal{K}_{[n]}$ we have

$$
\left\|\left(P_{[m]} J_{n}^{m}-J_{n}^{m} P_{[n]}\right) x\right\| \leq \varepsilon\left(\|x\|+\left\|P_{[m]} J_{n}^{m} x\right\|+\left\|P_{[n]} x\right\|\right)
$$

and the operator $P$ given by

$$
P J_{n} x=\lim _{m \rightarrow \infty} J_{m} P_{[m]} J_{n}^{m} x .
$$

For $J_{n} x \in \mathcal{K}_{\infty}$ is a well-defined orthogonal projection form $\mathcal{K}_{\infty}$ to $\mathcal{H}_{\infty}$.

Proof. In first step we notice that $\mathcal{D}\left(P_{[n]}\right)$, the domain of $P_{[n]}$, is the whole $\mathcal{K}_{[n]}$, so $\mathcal{D}\left(P_{[n]}\right)$ meets the condition $J_{n}^{m} \mathcal{D}\left(P_{[n]}\right) \subset \mathcal{D}\left(P_{[m]}\right)$ for all $n<m$. For $x \in \mathcal{K}_{[n]}$ and $m>n$, have k: $=(\mathbf{a}, \mathbf{b}) \in \mathbb{N}^{n} \times \mathbb{N}^{m-n}$ we have

$$
\begin{aligned}
P_{[n]} J_{n}^{m} x & =P_{[n]}\left(x \otimes e_{\{n, m\}}\right)=\sum_{\mathbf{k} \in \mathbb{N}^{m}}\left\langle x \otimes e_{\{n, m\}}, g_{\mathbf{k}}\right\rangle f_{\mathbf{k}}= \\
& =\sum_{(\mathbf{a}, \mathbf{b}) \in \mathbb{N}^{n} \times \mathbb{N}^{m-n}}\left\langle x \otimes e_{\{n, m\}}, g_{\mathbf{a}} \otimes g_{\mathbf{b}}\right\rangle f_{\mathbf{a}} \otimes f_{\mathbf{b}}= \\
& =\left\langle\sum_{\mathbf{a} \in \mathbb{N}^{n}}\left\langle x, g_{\mathbf{a}}\right\rangle f_{\mathbf{a}}\right\rangle \otimes\left\langle\sum_{b \in \mathbb{N}^{m-n}}\left\langle e_{\{n, m\}}, g_{\mathbf{b}}\right\rangle f_{\mathbf{b}}\right\rangle= \\
& =\left\langle\sum_{\mathbf{a} \in \mathbb{N}^{n}}\left\langle x, g_{\mathbf{a}}\right\rangle f_{\mathbf{a}}\right\rangle \otimes P_{\{n, m\}} e_{\{n, m\}} \doteq P_{[n]} x \otimes e_{\{n, m\}}=J_{n}^{m} P_{[n]} x .
\end{aligned}
$$

The equality $(\dot{\doteq})$ holds because $e_{\{n, m\}} \in \mathcal{H}_{\{n, m\}}$ and $P_{\{n, m\}}$ is an orthogonal projection onto $\mathcal{H}_{\{n, m\}}$. Now we write

$$
\left\|\left(P_{[m]} J_{n}^{m}-J_{n}^{m} P_{[n]}\right) x\right\|=\left\|\left(J_{n}^{m} P_{[n]}-J_{n}^{m} P_{[n]}\right) x\right\|=0 .
$$

The operator $P: \mathcal{K}_{\infty} \rightarrow \mathcal{K}_{\infty}$ is well defined by Theorem 2 . 
If $x \in \mathcal{K}_{\infty}$ then there exists $x_{n} \in \mathcal{K}_{[n]}$ for some $n \in \mathbb{N}$ such that $x=J_{n} x_{n}$. Moreover, for $m>n$ we have

$$
P x=\lim _{m \rightarrow \infty} J_{n} P_{[m]} J_{n}^{m} x_{n}=\lim _{m \rightarrow \infty} J_{m} J_{n}^{m} P_{[n]} x_{n}=\lim _{m \rightarrow \infty} J_{n} P_{[n]} x_{n}=J_{n} P_{[n]} x_{n} \in \mathcal{H}_{\infty} .
$$

From this we deduce that the range $P\left(\mathcal{K}_{\infty}\right)$ of $P$ equals $\mathcal{K}_{\infty}$.

For $x, y \in \mathcal{K}_{\infty}$ there exist $x_{n} \in \mathcal{K}_{[n]}, y_{k} \in \mathcal{K}_{[k]}$ such that $x=J_{n} x_{n}, y=J_{n} y_{k}$. Without lost of the generality we assume that $k \leq n$. Then

$$
\begin{aligned}
\langle P x, y\rangle & =\left\langle P J_{n} x_{n}, J_{k} y_{k}\right\rangle=\left\langle J_{n} P_{[n]} x_{n}, J_{k} y_{k}\right\rangle=\left\langle P_{[n]} x_{n} \otimes e_{\{n, \infty\}}, J_{k}^{n} y_{k} \otimes e_{\{n, \infty\}}\right\rangle= \\
& =\left\langle P_{[n]} x_{n}, J_{k}^{n} y_{k}\right\rangle\left\|e_{\{n, \infty\}}\right\|^{2}=\left\langle x_{n}, P_{[n]} J_{k}^{n} y_{k}\right\rangle\left\|e_{\{n, \infty\}}\right\|^{2}= \\
& =\left\langle x_{n}, J_{k}^{n} P_{[k]} y_{k}\right\rangle\left\|e_{\{n, \infty\}}\right\|^{2}=\left\langle x_{n} \otimes e_{\{n, \infty\}}, J_{k}^{n} P_{[k]} y_{k} \otimes e_{\{n, \infty\}}\right\rangle= \\
& =\left\langle J_{n} x_{n}, J_{k} P_{[k]} y_{k}\right\rangle=x, P y
\end{aligned}
$$

and

$$
P^{2} x=P^{2} J_{n} x_{n}=P\left(J_{n} P_{[n]} x_{n}\right)=J_{n} P_{[n]}^{2} x_{n}=J_{n} P_{[n]} x_{n}=P J_{n} x_{n}=P x .
$$

We have $P=P^{2}=P^{*}$ so $P$ is the orthogonal projection from $\mathcal{K}_{\infty}$ onto $\mathcal{H}_{\infty}$.

\section{Operators on Segal-Bargmann spaces}

In this section we briefly recall some informations about Segal-Bargmann spaces. Let us denote by

$$
\mu(z):=\frac{\alpha}{\pi} \exp \left(-\alpha|z|^{2}\right) \lambda(z)
$$

the one-dimensional Gaussian measure on $\mathrm{C}$. Here $\alpha$ is some positive parameter and $\lambda$ denotes the standard Lebesgue measure on $\mathbb{C}$. For $d \in \mathbb{N}$ the $d$-dimensional Gaussian measure is given by $\mu_{d}(z):=\bigotimes_{p=1}^{d} \mu\left(z_{p}\right)$, where $\mathbf{z}=\left(z_{1}, \ldots, z_{p}\right) \in \mathbb{C}^{d}$. If we take a scalar product $\langle f, g\rangle:=\int_{\mathbb{C}} f(\mathbf{z}) \overline{g(\mathbf{z})} d \mu_{d}(\mathbf{z})$ and the norm $\|f\|:=\sqrt{\langle f, g\rangle}$, then the space of the equivalence classes of all functions having this norm finite is a Hilbert space denoted by $L^{2}\left(\mathbb{C}^{d}, \mathrm{~d} \mu_{d}\right)\left(=L_{d}^{2}\right.$ for short $)$. The equivalence here means equality almost everywhere (i.e. except some set having zero measure). In some cases, the choice of representatives from such equivalence classes becomes obvious and this is so for analytic functions. If the domain of an analytic function is the whole $\mathbb{C}^{d}$, we call it an entire function. 
Definition 9. The set of all entire functions of $d$ complex variables that are square-integrable with respect to $\mathrm{d} \mu_{d}$ is called the Segal-Bargmann space $F_{d}$.

The $F_{d}$ space is a closed subspace in $L_{d}^{2}$.

Definition 10. If a function $\varphi: \mathbb{C} \rightarrow \mathbb{C}$ is $\mu$-measurable and $P$ denotes the orthogonal projection from $L^{2}$ onto $F_{1}$, then the operator defined by

$$
T_{\varphi}: F_{1} \supset \mathcal{D}\left(T_{\varphi}\right) \text { э } f \mapsto P(\varphi f) \in F_{1}
$$

on the set $\mathcal{D}\left(T_{\varphi}\right):=\left\{f \in F_{1}: \varphi f \in L^{2}\right\}$ is called Toeplitz operator and the function $\varphi$ is called the symbol of this operator $T_{\varphi}$.

From now we take $\mathcal{K}_{n}=L^{2}(\mathrm{C}, \mathrm{d} \mu), \mathcal{H}_{n}=F_{1}$. Using the notation of (6) we have $\mathcal{K}_{[n]}=L_{n}^{2}$ and $\mathcal{H}_{[n]}=F_{n}$.

It will be useful to write the following (isometric) identifications of tensor products:

$$
L_{n}^{2} \otimes L_{m}^{2}=L_{n+m}^{2}, \quad F_{n} \otimes F_{m}=F_{n+m} .
$$

Now we define the isometric embeddings

$$
J_{n}^{n+1}: L_{n}^{2} \ni f \mapsto f \otimes 1 \in L_{n+1}^{2}
$$

where $f \in L_{n}^{2}$ and $\mathbf{1}$ is the constant function $\mathbf{1}(\mathrm{z})=1, z \in \mathbb{C}$. We can construct two pairs of the isometric direct systems $\left\langle L_{n}^{2}, J_{n}^{m}\right\rangle,\left\langle F_{n}, J_{n}^{m}\right\rangle$ and the direct limits for them. We notice that $\lim _{\rightarrow} F_{n}$ is closed subspace in $\lim _{\rightarrow} L_{n}^{2}$.

Let us introduce the following convenient notations

$$
\begin{aligned}
& L_{\infty}^{2}:=\bigcup_{n \in \mathbb{N}} J_{n} L_{n}^{2}, \quad \mathbb{L}^{2}:=\lim _{\rightarrow} L_{n}^{2}, \\
& F_{\infty}:=\bigcup_{n \in \mathbb{N}} J_{n} F_{n}, \quad \mathbb{F}:=\lim _{\rightarrow} F_{n} .
\end{aligned}
$$

Recall that $\mathrm{L}^{2}$ and $\mathrm{F}$ are the completions of $L_{\infty}^{2}$, resp. $F_{\infty}$. Explicit form of the isometric embedding for above direct system is as follows

$$
\begin{aligned}
& J_{n}^{m}: L_{n}^{2} \ni f \mapsto f \otimes 1_{\{n, m\}}=f \otimes \bigotimes_{k=n+1}^{m} 1 \in L_{m}^{2}, \\
& J_{n}: L_{n}^{2} \ni f \mapsto f \otimes 1_{\{n, \infty\}}=f \otimes \bigotimes_{k=n+1}^{\infty} 1 \in \mathbb{L}^{2} .
\end{aligned}
$$

In Janas and Rudol (1990, 1994, 1995) the authors studied bounded operators that are some generalization of Toeplitz operators acting on functions of finitely 
many variables. They used two non-equivalent approaches, direct limit and "measure-theoretic". For the direct limit they give some conditions for the compactness of Toeplitz operators in contrast to the non-existence of nonzero compact Toeplitz operators in the second approach.

The idea of constructing the Segal-Bargmann(-Fock) space of infinitely many variables via direct limits is due to Segal (1978). In the case of functions of finitely many variables, Toeplitz operators on $F_{d}$ were used by by Berger and Coburn (1986, 1987) to represent observables from quantum mechanics.

Now we define Toeplitz operators on the direct limit $\mathbb{F}$. We use an admissible family of frames to obtain convenient form of the orthogonal projection $P$ form $L^{2}$ onto $\mathbb{F}$ (Theorem 8). This is a preparation for the future study of properties of these operators.

By $L_{k}^{\infty}$ we denote the function space of all $k$-variable essentially bounded $\mu_{k}$-measurable functions. Recall that the essential supremum which serves as a norm for a function $\varphi$ in this space is given by

$$
\|\varphi\|_{\infty}=\inf \left\{C \geq 0:|\varphi(z)| \leq C \text { for } \mu_{k} \text {-a.e. } z \in \mathbb{C}^{k}\right\} .
$$

Using isometric embeddings like (9) we can construct isometric direct system $\left\langle L_{n}^{\infty}, J_{n}^{m}\right\rangle$ with $L_{\infty}^{\infty}:=\bigcup_{n \in \mathbb{N}} J_{n} L_{n}^{\infty}$ and $\mathbb{L}^{\infty}:=\lim _{\rightarrow} L_{n}^{\infty}$.

Definition 11. If $\varphi_{k} \in L_{k}^{\infty}$ then $\varphi=J_{k} \varphi_{k} \in L_{\infty}^{\infty}$ and the Toeplitz operator with symbol $\varphi$ is given by

$$
T_{\varphi}: \mathbb{F} \supset \mathcal{D}\left(T_{\varphi}\right) \ni f \mapsto P(\varphi f) \in \mathbb{F}
$$

where $f \in \mathcal{D}\left(T_{\varphi}\right):=\left\{f \in \mathbb{F}: \varphi f \in \mathbb{L}^{2}\right\}(=\mathbb{F})$.

We write how the operator $T_{\varphi}$ acts on $f \in \mathbb{F}$ in some cases. If $f \in F_{\infty}$ then there exists $f_{n} \in F_{n}$ such that $f=J_{n} f_{n}$. Now we have for $n<k$.

$$
T_{\varphi} f=P\left(J_{k} \varphi_{k} J_{n} f_{n}\right)=P\left(J_{k}\left(\varphi_{k} J_{n}^{k} f_{n}\right)\right)=J_{k} P_{[k]}\left(\varphi_{k} J_{n}^{k} f_{n}\right)=J_{k} T_{\varphi_{k}}\left(J_{n}^{k} f_{n}\right)
$$

and for $n \geq k$

$$
T_{\varphi} f=P\left(J_{k} \varphi_{k} J_{n} f_{n}\right)=J_{n} P_{[n]}\left(J_{k}^{n} \varphi_{k} f_{n}\right)=J_{n} T_{J_{k}^{n} \varphi_{k}} f_{n} .
$$

If $f \in \mathbb{F} \mid F_{\infty}$ then exists a sequence $\left\{f_{n}\right\} \subset F_{\infty}$ such that $\lim _{n \rightarrow \infty}\left\|f-f_{n}\right\|=0$. Hence, we can estimate

$$
\left\|T_{\varphi}\left(f-f_{n}\right)\right\|=\| P\left(\varphi\left(f-f_{n}\right)\|\leq\| \varphi\|\| f-f_{n} \| \stackrel{n \rightarrow \infty}{\longrightarrow} 0\right.
$$

and we have $T_{\varphi} f=\lim _{n \rightarrow \infty} T_{\varphi} f_{n}$. 
Definition 12. If $\varphi \in \mathbb{L}^{\infty} \backslash L_{\infty}^{\infty}$ then there exists a sequence $\left\{\varphi_{k}\right\} \subset L_{\infty}^{\infty}$ such that $\lim _{k \rightarrow \infty}\left\|\varphi-\varphi_{k}\right\|=0$. Toeplitz operator with symbol $\varphi$ is defined by

$$
T_{\varphi} f:=\lim _{k \rightarrow \infty} T_{\varphi_{k}} f, \quad f \in \mathbb{F} .
$$

This operator is well defined because for all $f \in \mathbb{F}$ we have the following estimate

$$
\left\|T_{\varphi_{k}} f-T_{\varphi_{m}} f\right\| \leq\left\|\left(\varphi_{k}-\varphi_{m}\right) f\right\| \leq\left\|\varphi_{k}-\varphi_{m}\right\|\|f\| \leq \varepsilon\|f\| .
$$

So, we notice that the sequence $\left\{T_{\varphi_{k}} f\right\}$ is convergent and the limit does not depend on the choice of particular $\left\{\varphi_{k}\right\}$ approximating $\varphi$.

Theorem 13. Let $\varphi \in L_{\infty}^{\infty}$, so that $\varphi=J_{k} \varphi_{k}$ for some $\varphi_{k} \in L_{k}^{\infty}$. If $T_{\varphi_{k}} \neq 0$ then $T_{\varphi}$ cannot be compact.

Proof. By contradiction we assume that $T_{\varphi}$ is a compact operator. If we take any sequence $\left\{f_{n}\right\} \subset \mathbb{F}$ weakly convergent to 0 then there should be $\left\|T_{\varphi} f_{n}\right\| \stackrel{n \rightarrow \infty}{\longrightarrow} 0$. So, we take a sequence of vectors of the form $f_{n}:=J_{k+1}\left(g \otimes \widetilde{f_{n}}\right)$, where $g \in F_{k}, \widetilde{f_{n}} \in F_{1}$. What is more we assume that $T_{\varphi_{k}} g \neq 0$ and $\widetilde{f_{n}}$ is weakly convergent to $0\left(\widetilde{f_{n}} \rightarrow 0\right)$ but it is not norm convergent. From this we have $f_{n} \rightarrow 0$. Now we write the following equalities

$$
\begin{aligned}
\left\|T_{\varphi} f_{n}\right\| & =\left\|P\left(J_{k} \varphi_{k} \cdot J_{k+1}\left(g \otimes \widetilde{f_{n}}\right)\right)\right\|=\left\|P J_{k+1}\left(J_{k}^{k+1} \varphi_{k} \cdot\left(g \otimes \widetilde{f_{n}}\right)\right)\right\|= \\
& =\left\|J_{k+1} P_{[k+1]}\left(\varphi_{k} \otimes 1\right)\left(g \otimes \widetilde{f_{n}}\right)\right\|=\left\|P_{[k]} \otimes P_{k+1}\left(\varphi_{k} \cdot g\right) \otimes\left(1 \cdot \widetilde{f_{n}}\right)\right\|= \\
& =\left\|P_{[k]}\left(\varphi_{k} \cdot g\right) \otimes P_{k+1}\left(\widetilde{f_{n}}\right)\right\|=\left\|T_{\varphi_{k}} g \widetilde{f_{n}}\right\| .
\end{aligned}
$$

The first term $\left\|T_{\varphi_{k}} g\right\|$ is constant (non-zero) and the second term $\left\|\widetilde{f}_{n}\right\|$ is not convergent to 0 so $\left\|T_{\varphi} f_{n}\right\|$ do not converge to 0 which is a contradiction with assumed compactness of the operator $T_{\varphi}$. This ends the proof.

The author wants to express his gratitude to professor Krzysztof Rudol for conversations and numerous comments that helped write and improve this article.

\section{References}

Christensen O., 2008, Frames and Bases an Introductory Course, Birkhauser, Boston. Berger C.A., Coburn L.A., 1986, Toeplitz operators and quantum mechanics, Journal of

Functional Analysis, vol. 68, pp. 273-299. https://doi.org/10.1016/0022-1236(86)90099-6. Berger C.A., Coburn L.A., 1987, Toeplitz operators on the Segal-Bargmann space, Transactions of the American Mathematical Society, vol. 301, pp. 813-829. https:/ /doi.org/10.2307/2000671. 
Janas J., 1995, Inductive limit of Operators and its Applications, Studia Mathematica, t. 90, pp. 87-102. https://doi.org/10.4064/sm-90-2-87-102.

Janas J., Rudol K., 1990, Toeplitz operators on the Segal-Bargmann space of infinitely many variables, [in:] Helson H., Sz.-Nagy B., Vasilescu F.-H., Arsene G. (eds.), Linear Operators in Function Spaces, Operator Theory: Advances and Applications, Birkhäuser Basel, pp. 217-228. https://doi.org/10.1007/978-3-0348-7250-8_15.

Janas J., Rudol K., 1994, Two Approaches to Toeplitz Operators on Fock Space, [in:] Antoine J.P., Ali S.T., Lisiecki W., Mladenov I.M., Odzijewicz A. (eds.), Quantization and Infinite-Dimensional Systems, Springer, Boston, MA, pp. 3-7. https:// doi.org/10.1007/978-1-4615-2564-6_1.

Janas J., Rudol K., 1995, Toeplitz Operators in Infinitely Many Variables, [in:] Gheondea A., Strătilă Ş., Timotin D. (eds.), Topics in Operator Theory, Operator Algebras and Applications: 15th International Conference on Operator Theory, Timisoara (Romania), June 6-10, 1994, IMAR, Bucharest, pp. 147-160.

Marčenko A.V., 1974, On Inductive Limits of Linear Spaces and Operators and Their Applications, Moscow University Bulletin, no. 2, pp. 26-33 [Марченко A.B., 1974, Об индуктивных пределах линейных пространстви операторов и их приложениях, Ве́стник Моско́вского Университе́та, № 2, с. 26-33].

Marčenko A.V., 1975, Selfadjoint Differential Operators with an Infinite Number of Independent Variables, Mathematics of the USSR-Sbornik, vol. 25, no. 2, pp. 259-275 [Марченко А.В., 1975, Самосопряженные дифференциальные операторы с бесконечным числом независимых переменных, Математический сборник, Т. 25, № 2 , c. 259-275].

Rudol K., 1992, Conditionally convergent tensor products, Integral Equations and Operator Theory, vol. 15, pp. 697-705. https://doi.org/10.1007/BF01195784.

Segal I.E., 1978, The complex wave representation of the free boson field, [in:] Gohberg I., Kac M. (eds.), Topics in functional analysis: essays dedicated to M. G. Krein on the occasion of his 70th birthday, Advances in Mathematics: Supplementary Studies, vol. 3, Academic Press, New York, pp. 321-344. 